Document downloaded from:

http://hdl.handle.net/10251/156938

This paper must be cited as:

Samaniego, D.; Vidal Rodriguez, B. (2019). Brillouin Microwave Filter with enhanced Skirt Selectivity using a Birefringent Fiber. IEEE Photonics Technology Letters. 31(6):431-434. https://doi.org/10.1109/LPT.2019.2897398

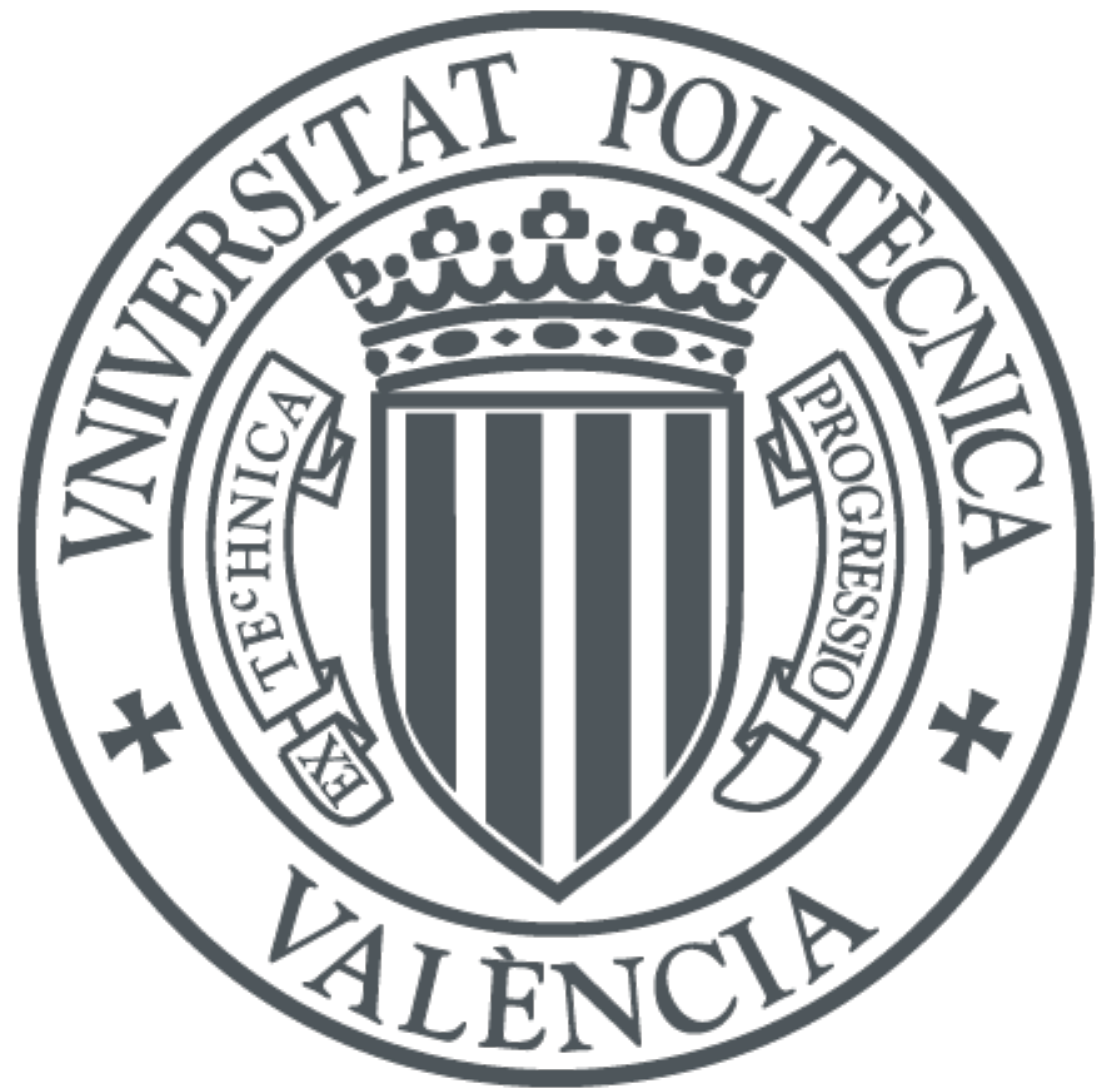

The final publication is available at

https://doi.org/10.1109/LPT.2019.2897398

Copyright Institute of Electrical and Electronics Engineers

Additional Information

(C) 2019 IEEE. Personal use of this material is permitted. Permissíon from IEEE must be obtained for all other uses, in any current or future media, including reprinting/republishing this material for advertisíng or promotional purposes, creating new collective works, for resale or redistribution to servers or lists, or reuse of any copyrighted component of this work in other works. 


\title{
Brillouin Microwave Filter with enhanced Skirt Selectivity using a Birefringent Fiber
}

\author{
Diego Samaniego, Member, IEEE and Borja Vidal, Senior Member, IEEE
}

\begin{abstract}
A technique to enhance the slope of a photonic microwave filter based on stimulated Brillouin scattering is proposed. It relies on exploiting the polarization dependence of Brillouin gain in birefringent fibers. The presence of two orthogonal Brillouin gains/loss in birefringent fibers results in two filter responses that can be subtracted in a balanced photodetector to remove the slow Lorentzian decay of the natural Brillouin gain response. Experimental results show that a filter slope of 8.3 dB/oct can be obtained
\end{abstract}

Index Terms - Microwave photonics, scattering, fiber nonlinear optics, nonlinear effects.

\section{INTRODUCTION}

Among the different areas of microwave photonics, filtering has attracted considerable attention [1] due to its potential to offer an alternative implementation of microwave filters with very large bandwidth, tunability and reconfigurability. Several approaches are being investigated such as the use of integrated photonic circuits [2-4] or the exploitation of nonlinear effects such as Four-Wave Mixing [5] and, especially, Stimulated Brillouin Scattering (SBS) [6-11]. SBS is very appealing for microwave filtering because it can be used to implement narrow filter responses with tuning and reconfiguration capabilities. Additionally, this effect can be stimulated in integrated circuits which is critical for cost reduction [11]. Finally, several Brillouin responses can be combined to implement flat-top filter responses [10].

Although SBS-based filters with good shape factors have been demonstrated [10], it is done by increasing the filter bandwidth since the skirt selectivity of SBS-based photonic microwave filters is given by the slow-decaying Lorentzian tail of the natural Brillouin response, which produces filters with poor rejection of close adjacent channels.

Recently, a method to enhance the selectivity of SBS-based photonic microwave filters has been proposed [12] which is based on combining a gain response with two frequency-offset loss ones that are used to attenuate the tails of the Lorentzian response. However, the need of three pump waves, i.e. three microwave oscillators, increases system complexity and make tunability more difficult.

Here, an alternative approach for improving skirt selectivity while keeping easy tunability is explored. It relies on photonic phenomena to simplify the architecture. In particular, it uses the usually deleterious effect of the double Brillouin gain/loss response happening in low birefringent optical fibers [13].

\section{PRINCIPLE OF OPERATION}

Considering a birefringent fiber and assuming operation in the undepleted pump regimen, the SBS amplification can be seen as composed of two SBS gain terms [13]: maximum gain $\left(G_{\max }\right)$ and minimum gain $\left(G_{\min }\right)$, with orthogonal states of polarization (SOP). The SOP of the maximum gain is parallel to the conjugate of the pump wave $\left(\overline{E_{p}^{*}}\right)$, whilst the SOP of the minimum gain is orthogonal to the conjugate of the pump wave, as shown in Fig. 1a. Similarly, SBS attenuation has two terms: maximum loss $\left(A_{\max }\right)$ and minimum loss $\left(A_{\min }\right)$.

If the SOP of a phase-modulated optical carrier is aligned at $45^{\circ}$ in relation to the conjugate of the pump wave, the signal components on each of the orthogonal axis will experience maximum and minimum SBS gain/loss respectively, as shown in Fig. 1a for the case of SBS gain.

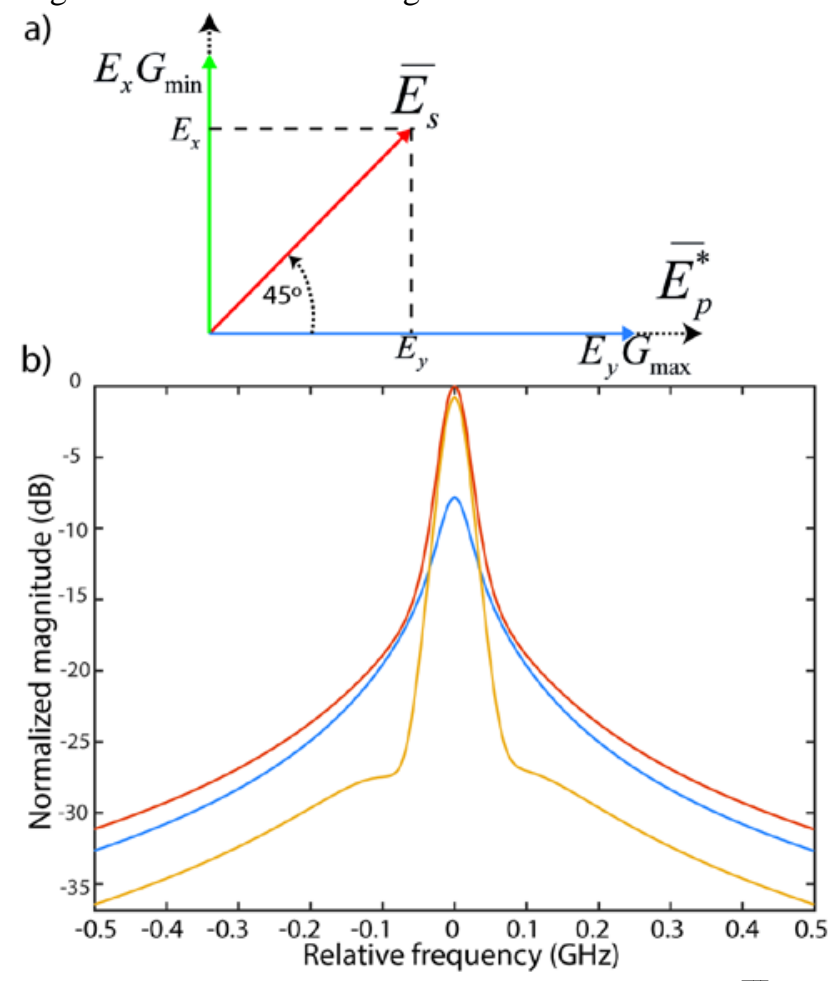

Fig. 1. (a) Amplified signal on maximum and minimum gain. $\overline{E_{s}}$ is the field of the signal wave before SBS gain. $G_{\max }$ and $G_{\min }$ are the maximum and minimum Brillouin gain in a birefringent fiber. (b) Principle of operation. Frequency response of maximum (red) and minimum (blue) SBS gain and combined response at the output of the detector (yellow).

Thus, each orthogonal polarization carries a copy of the same microwave-modulated optical signal but with a different SBS gain. It is possible to split the signals $E_{y} G_{\max }$ and $E_{x} G_{\min }$ 
using a polarization beam splitter. If the relative amplitude between these two responses $\left(E_{y} G_{\text {max }}\right.$ and $\left.E_{x} G_{\text {min }}\right)$ is adjusted, they can be subtracted using a balanced photodiode to suppress the tails of the SBS gain, as shown in Fig. 1b. Thus, a considerable reduction of the decaying tails can be obtained while having a small effect on the passband loss.

\section{THEORY}

Assuming no pump depletion and that the losses in the fiber are low, the SBS gain and loss induced by a counterpropagating pump can be described as follows [14],

$$
\begin{aligned}
g(f c+f)= & \frac{g_{o} P_{p}}{A_{e f f}} \frac{\left(\frac{\Delta v_{B}}{2}\right)^{2}}{\left(-f_{p}+v_{B}+f\right)^{2}+\left(\frac{\Delta v_{B}}{2}\right)^{2}}+ \\
& j \frac{g_{o} P_{p}}{2 A_{e f f}} \frac{\Delta v_{B}\left(-f_{p}+v_{B}+f\right)}{\left(-f_{p}+v_{B}+f\right)^{2}+\left(\frac{\Delta v_{B}}{2}\right)^{2}} \\
\alpha(f c-f)= & -\frac{g_{o} P_{p}}{A_{e f f}} \frac{\left(\frac{\Delta v_{B}}{2}\right)^{2}}{\left(-f_{p}+v_{B}+f\right)^{2}+\left(\frac{\Delta v_{B}}{2}\right)^{2}}+ \\
& j \frac{g_{o} P_{p}}{2 A_{e f f}} \frac{\Delta v_{B}\left(-f_{p}+v_{B}+f\right)}{\left(-f_{p}+v_{B}+f\right)^{2}+\left(\frac{\Delta v_{B}}{2}\right)^{2}}
\end{aligned}
$$

where $f_{c}$, and $f_{p}$ represent the frequency of the optical carrier and pump wave respectively; $v_{B}$ is the Brillouin frequency shift, $\Delta v_{B}$ represents the Brillouin linewidth of the fiber; $\mathrm{g}_{0}$ is the Brillouin gain factor; and $P_{p}$ is the pump power.

Under small signal condition, the optical field of the forward-propagating RF-modulated signal by a phase modulator (PM) is given by [15]:

$$
\overline{E_{s}}=E_{0} e^{i 2 \pi f_{c} t}\left[J_{0}(m)+J_{1}(m) e^{i 2 \pi f_{R F} t}-J_{1}(m) e^{-i 2 \pi f_{R F} t}\right]
$$

where $E_{0}$ represents the optical field at the input of the external modulator, $J_{n}$ denotes the nth-order Bessel function of the first kind with $n=0, \pm 1, m$ is the phase modulation index, and $f_{R F}$ represents the frequency of the input microwave signal.

The Jones vector of the optical field after SBS is:

$$
\overrightarrow{E_{s}^{\prime}}=\left[\begin{array}{l}
E_{x} S B S_{\min } \\
E_{y} S B S_{\max }
\end{array}\right]
$$

where $E_{y}$ and $E_{x}$ represents the signal wave projection on the axes of the birefringent fiber, and $S B S_{\max }, S B S_{\min }$ are the maximum and minimum SBS gain/loss, respectively.

$$
\begin{aligned}
E_{X} S B S_{\min } & =\frac{E_{0} e^{i 2 \pi f_{c} t}}{\sqrt{2}}\left[J_{0}(m)+J_{1}(m) e^{i 2 \pi f_{R F} t} e^{g\left(f_{c}+f_{R F}\right) L \zeta}\right. \\
& \left.-J_{1}(m) e^{-i 2 \pi f_{R F} t} e^{\alpha\left(f_{c}-f_{R F}\right) L \zeta}\right]
\end{aligned}
$$

$$
\begin{aligned}
E_{y} S B S_{\max } & =\frac{E_{0} e^{i 2 \pi f_{c} t}}{\sqrt{2}}\left[J_{0}(m)+J_{1}(m) e^{i 2 \pi f_{R F} t} e^{g\left(f_{c}+f_{R F}\right) L \zeta}\right. \\
& \left.-J_{1}(m) e^{-i 2 \pi f_{R F} t} e^{\alpha\left(f_{c}-f_{R F}\right) L \zeta}\right]
\end{aligned}
$$

where the term $\zeta$ is $2 / 3$ for the maximum gain/loss and $1 / 3$ for the minimum gain/loss in birefringent fiber [13]. The magnitude and phase of SBS gain/loss response due to SBS can be expressed as:

$$
\begin{gathered}
G_{\text {max,min }}\left(f_{R F}\right)=e^{\operatorname{Re}\left(g\left(f_{c}+f_{R F}\right) L \zeta\right.} \\
A_{\text {max,min }}\left(f_{R F}\right)=e^{\operatorname{Re}\left(\alpha\left(f_{c}-f_{R F}\right) L \zeta\right.} \\
\varphi_{\text {max,min }}\left(f_{R F}\right)=\operatorname{Im}\left(g\left(f_{C}+f_{R F}\right) L \zeta\right)=\operatorname{Im}\left(\alpha\left(f_{C}-f_{R F}\right) L \zeta\right)
\end{gathered}
$$

To enhance out-of-band rejection, the signal to be filtered is modulated in phase and after the SBS gain/loss process in the birefringent optical fiber, the signal is passed through a polarization beam splitter (PBS), separating the optical field of the signal in two signal waves, $E_{y} S B S_{\max }$ and $E_{x} S B S_{\min }$. Fig. 2a shows the signal experiencing maximum gain/loss and Fig. $2 \mathrm{~b}$ the signal with minimum gain/loss.

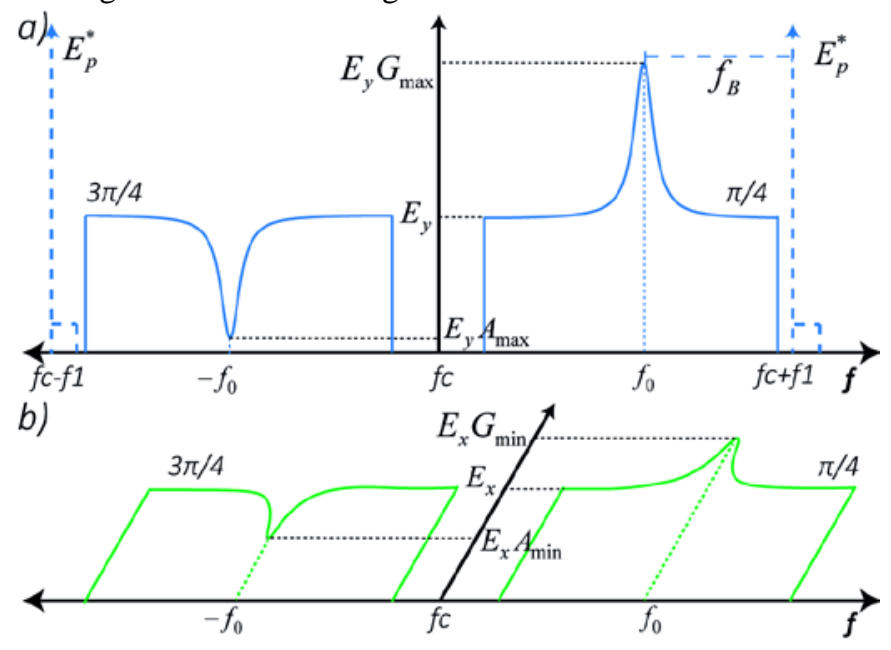

Fig. 2. Phase-modulated optical signal experiencing SBS. (a) Maximum gain/loss, $E_{y} S B S_{\max }$ and (b) Minimum gain/loss, $E_{x} S B S_{\min } . f_{c} \pm f_{1}$ are the frequencies of the pump waves.

Omitting the DC component as well as higher-order harmonics, the RF power after photodetection of the two optical signals $\left(P_{\text {RFout }}, P_{\text {RFout }}\right)$ is:

$$
\begin{aligned}
& \underset{\max }{P_{R F o u t}} \propto\left[\left(E_{y} S B S_{\max }\right) *\left(E_{y} S B S_{\max }\right)^{*}\right]^{2} \\
& \propto\left[\left|G_{\text {max }}\left(f_{R F}\right)\right|^{2}+\left|A_{\text {max }}\left(f_{R F}\right)\right|^{2}-2 \cos \left(2 \varphi_{\text {max }}\right)\right] \\
& \underset{\substack{R \text { mout } \\
P_{\text {min }}}}{ } \propto\left[\left(E_{x} S B S_{\min }\right) *\left(E_{x} S B S_{\min }\right)^{*}\right]^{2} \\
& \propto\left[\left|G_{\text {min }}\left(f_{R F}\right)\right|^{2}+\left|A_{\text {min }}\left(f_{R F}\right)\right|^{2}-2 \cos \left(2 \varphi_{\text {min }}\right)\right]
\end{aligned}
$$

Finally, the filter frequency response based on the SBS gain/loss process in birefringent optical fibers is expressed as, 


$$
\begin{aligned}
H\left(f_{R F}\right) & =\eta^{2} H\left(f_{R F}\right)_{\max }-H\left(f_{R F}\right)_{\min } \\
& \propto\left[\left(\eta^{2}\left|G_{\max }\left(f_{R F}\right)\right|^{2}-\left|G_{\min }\left(f_{R F}\right)\right|^{2}\right)+\right. \\
& \left(\eta^{2}\left|A_{\max }\left(f_{R F}\right)\right|^{2}-\left|A_{\min }\left(f_{R F}\right)\right|^{2}\right)- \\
& \left(2 \eta^{2} \cos \left(2 \varphi_{\max }\right)-2 \cos \left(2 \varphi_{\min }\right)\right]
\end{aligned}
$$

The factor $\eta$ is the optical attenuation that has to be applied to the signal experiencing maximum gain/loss (Fig. 2a) to match the amplitudes of both Brillouin response tails after each photodetector. Fig. 3 (left) shows the theoretical evolution of the slope of the filter response as a function $\eta$. For a value of $\eta=0 \mathrm{~dB}$, the filter response associated with the maximum SBS gain dominates if the pump power is high enough and the filter slope is the conventional value of around $4 \mathrm{~dB} / \mathrm{oct}$ from the natural Lorentzian response. Beyond a value of $\eta=3.1 \mathrm{~dB}$, lobes appear in the filter response that degrade out-of-band rejection. Fig. 3 (right) shows the additional microwave loss in the filter response induced by the attenuation $\eta$. The additional microwave loss follows a linear trend, falling as expected $2 \mathrm{~dB}$ for each optical $\mathrm{dB}$ until a factor around $\eta=1.5 \mathrm{~dB}$, where the effect of the subtraction between the two filter responses starts to dominate.

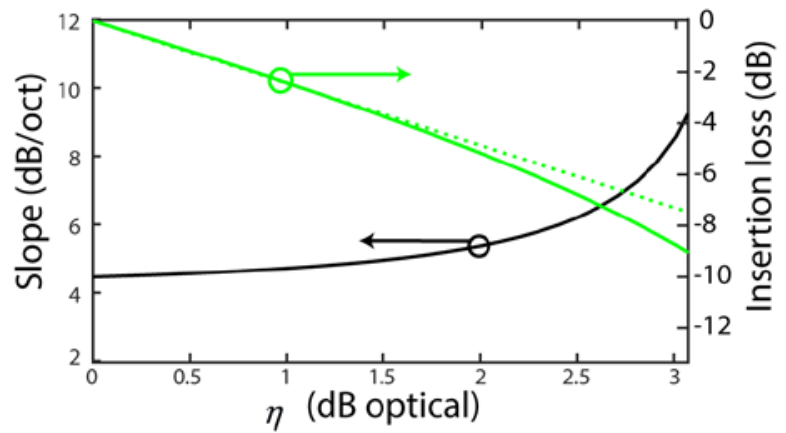

Fig. 3. Slope of the Brillouin-based photonic microwave filter (left) and microwave additional loss (right) versus factor $\eta$ for a pump signal of 12.5 $\mathrm{dBm}$ that induced a maximum SBS gain value of $\left|G_{\max }\left(f_{R F}\right)\right|^{2}=14 \mathrm{~dB}$.

\section{EXPERIMENTAL RESULTS}

Fig. 4 shows the experimental setup. The light from a DFB source at $1548 \mathrm{~nm}(12.5 \mathrm{dBm})$ is divided by a directional coupler 50/50. The upper path of the setup was used to generate the pump while the lower one corresponds to the signal.

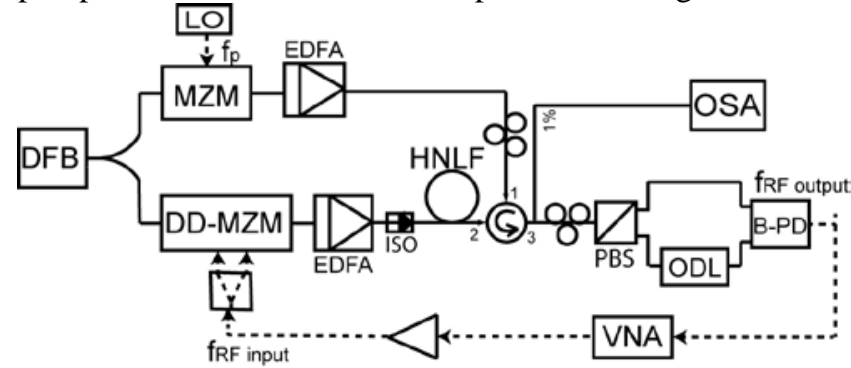

Fig. 4. Block diagram of the experimental setup. DFB: distributed feedback laser, MZM: Mach-Zehnder modulator, DD-MZM: Dual-Drive MZM, ISO: isolator, PBS: polarization bean splitter, ODL: optical delay line, B-PB: balanced photo detector, VNA: vector network analyzer, LO: local oscillator.

In the upper path, the optical signal is introduced in an MZM biased at the minimum transmission point (MITB), generating a DSB-SC modulation. The pump signal at $f_{c}+f_{1}$ induces an SBS gain response over the upper sideband and the pump at $f_{c}-f_{1}$ induces an SBS loss response to the lower sideband. Optical amplification is used to boost the pump signal and, finally, an optical circulator directs the pump toward the nonlinear medium (a reel of $1 \mathrm{~km}$-long HNLF with Brillouin linewidth $\Delta v_{B}=40 \mathrm{MHz}$ and Brillouin frequency shift $v_{B}=$ $9.64 \mathrm{GHz}$ ).

In the lower path, the data signal is phase modulated using a DD-MZM and a microwave hybrid coupler. The DD-MZM is biased at the maximum transmission point (MATB). An isolator (ISO) is used to eliminate the pump signal after it crossed through the HNLF. The modulated signal is boosted by optical amplification and it crosses the HNLF. Through an optical circulator, it reaches a polarization beam splitter (PBS). The SOP of the signal wave was adjusted to $45^{\circ}$ in relation to the maximum and minimum SBS gain axes by means of a polarization controller as was shown in Fig. 1a. Finally, both signals are detected by a balanced photodiode. The filter frequency response can be tuned by changing the frequency of the single local oscillator.

Fig. 5 shows the filter response measured for an SBS-based filter with phase modulation experiencing maximum (red) and minimum (green) SBS gain/loss and with the system described in Fig. 4. There is a good agreement between theory and experimental results.
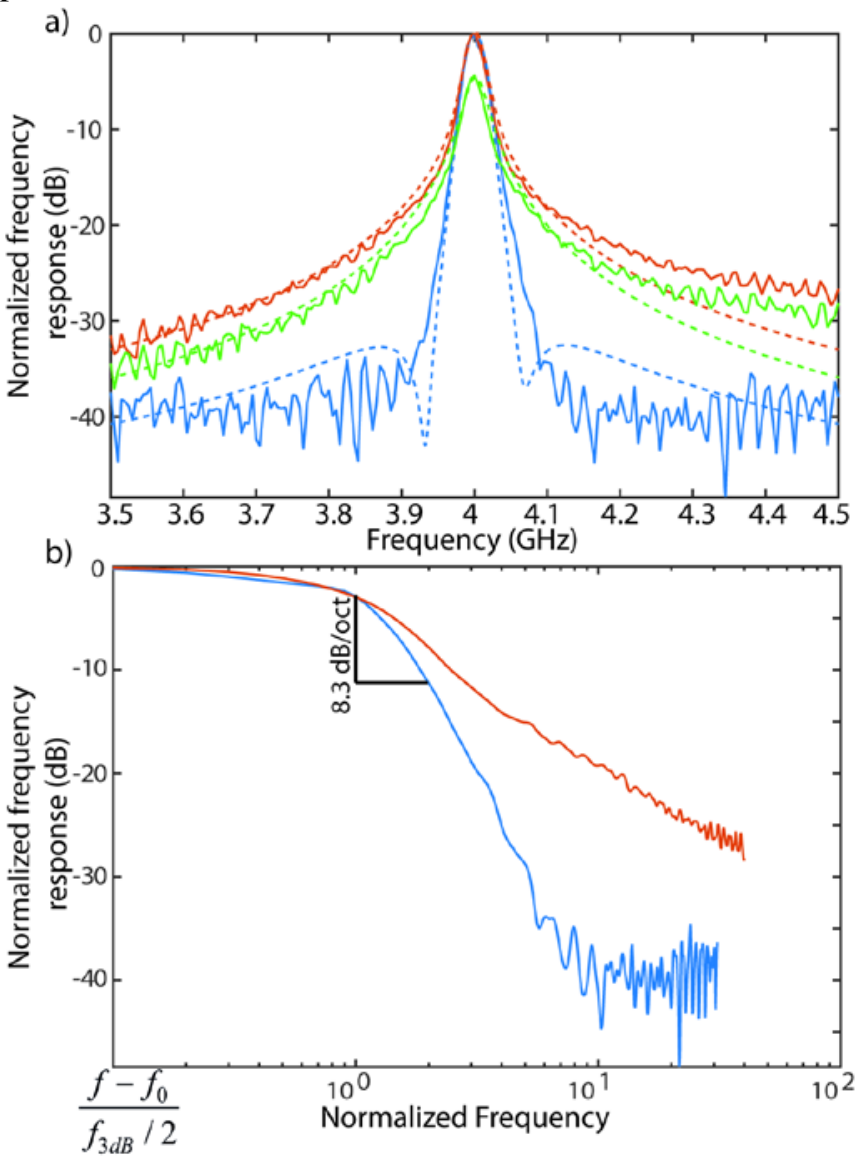

Fig. 5. (a) Experimental SBS-based filter response: with phase modulation and pump power of $12 \mathrm{dBm}$ experiencing maximum (red) and minimum (green) SBS gain/loss; SBS-based photonic microwave filter exploiting fiber birefringence and pump power of $14.5 \mathrm{dBm}$ (blue). Theoretical SBS-based filter response: with phase modulation experiencing maximum (red dotted) and minimum (green dotted) SBS gain/loss; SBS gain/loss process in presence of birefringent optical fiber in combination with balanced detection (blue dotted). (b) Bode diagram of the experimental SBS-based filter response. 
It can be seen in Fig. 5 that the slope of the Lorentzian Brillouin response is enhanced. The filter response shows a mean filter slope of $8.3 \mathrm{~dB} / \mathrm{oct}$ and an out-of-band rejection ratio of around $40 \mathrm{~dB}$. For the sake of comparison, a conventional SBS-based filter response of the same gain shows a slope of $4.8 \mathrm{~dB} / \mathrm{oct}$.

Fig. 6 compares in terms of bandwidth and shape factor a conventional SBS-based filter with phase modulation and the new technique proposed here for different pump powers. Fig. 6 a shows that the bandwidth at $-20 \mathrm{~dB}$ is considerably reduced with the new technique whilst the bandwidth at $-3 \mathrm{~dB}$ remains quite constant. Fig. $6 \mathrm{~b}$ shows that the filter shape factor is enhanced with the proposed method.

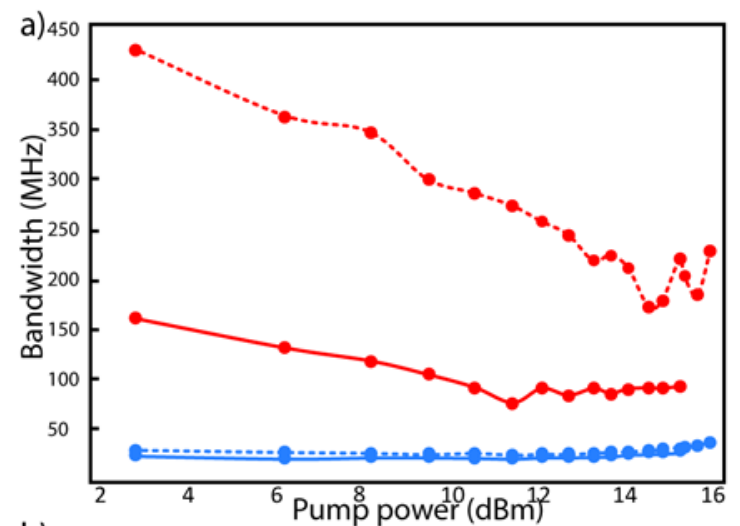

b)

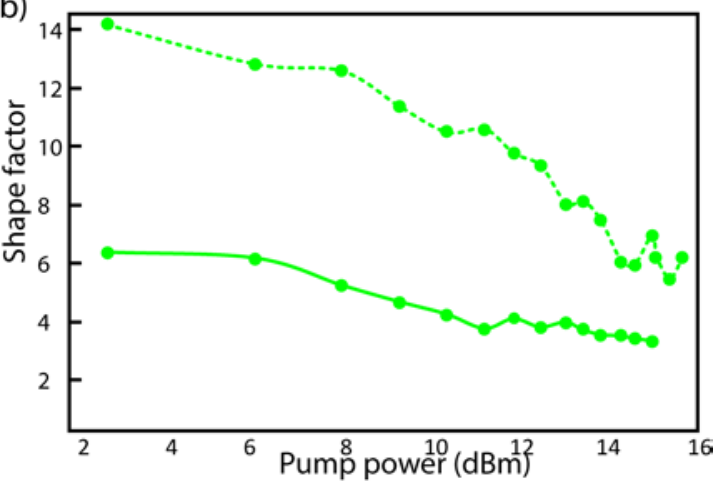

Fig. 6. Comparison between a conventional phase-modulated SBS microwave filter (dotted) and the new technique (solid). (a) Bandwidth of the filter response. At $-20 \mathrm{~dB}$ (red) and $-3 \mathrm{~dB}$ (blue). (b) Shape factor.

\section{CONCLUSION}

A method to enhance the slope of SBS-based photonic microwave filters has been proposed. By exploiting the usually deleterious effect induced by birefringent fiber on Brillouin processing it has been shown that maximum and minimum Brillouin gain/loss can be used to enhance the filter response. A filter with a slope of $8.3 \mathrm{~dB} /$ oct and an out-of-band rejection of $40 \mathrm{~dB}$ has been demonstrated. The filter selectivity is enhanced with a simple scheme and just a single stage and one pump wave.

\section{ACKNOWLEDGMENT}

D. Samaniego acknowledges funding from SENESCYT “Convocatoria Abierta 2012” (No, AR2Q-4233).

\section{REFERENCES}

[1] J. Yao, "Photonics to the Rescue: A Fresh Look at Microwave Photonic Filters.” IEEE Microwave Magazine, vol. 16, no. 8, pp. 46-60, 2015.

[2] M. Rasras, K.Y. Tu, D.M. Gill, Y.K. Chen, A.E. White, S.S. Patel, A. Pomerene, D. Carothers, J. Beattie, M. Beals, J. Michel, L.C. Kimerling, "Demonstration of a Tunable Microwave-Photonic Notch Filter Using Low-Loss Silicon Ring Resonators", J. Light. Technol., vol. 27, no. 12, p. 2105-2110, 15 June 2009.

[3] J. Palací, G.E. Villanueva, J.V. Galán, J. Martí, B. Vidal, “Single Bandpass Photonic Microwave Filter based on a Notch Ring Resonator", IEEE Photonics Technology Letters, vol. 22, no. 17, pp. 1276-1278, September 2010.

[4] Y. Liu, J. Hotten, A. Choudhary, B. J. Eggleton, D. Marpaung, “Alloptimized integrated RF photonic notch filter”, Optics Letters, vol. 42, no. 22, pp. 4631-4634, 15 November 2017.

[5] B. Vidal, J. Palací, J. Capmany, "Reconfigurable Photonic Microwave Filter based on Four-Wave Mixing”, IEEE Photonics Journal, vol. 4, no. 3, pp. 759-764, June 2012.

[6] B. Vidal, M. A. Piqueras, J. Martí, "Tunable and Reconfigurable Photonic Microwave Filter based on Stimulated Brillouin Scattering”, Optics Letters, vol. 32, no. 1, pp. 23-25, 1 January 2007.

[7] B. Vidal, T. Mengual, J. Marti, "Photonic Microwave Filter with Single Bandpass Response based on Brillouin Processing and SSB-SC", IEEE International Topical Meeting on Microwave Photonics (MWP2009), Valencia (Spain), 14-16 October 2009.

[8] W. Zhang and R. A. Minasian, "Switchable and tunable microwave photonic brillouin-based filter," IEEE Photonics J., vol. 4, no. 5, pp. 1443-1455, 2012.

[9] Y. Stern, K. Zhong, T. Schneider, R. Zhang, Y. Ben-Ezra, M. Tur, A. Zadok, "Tunable sharp and highly selective microwave-photonic bandpass filters based on stimulated Brillouin scattering”, Photon. Res., vol. 2, no 4, p. B18-B25, 2014.

[10] W. Wei, L. Yi, Y. Jaouën, M. Morvan, "Brillouin rectangular optical filter with improved selectivity and noise performance", IEEE Photonics Technology Letters, vol. 27, no 15, pp. 1593-1596, 2015.

[11] D. Marpaung, B. Morrison, M. Pagani, R. pant, D.Y. Choi, B. LutherDavies, S. J. Madden, B. J. Eggleton, "Low-power, chip-based stimulated Brillouin scattering microwave photonic filter with ultrahigh selectivity", Optica, vol. 2, no. 2, pp. 76-83, February 2015.

[12] D. Samaniego, B. Vidal, "Photonic microwave filter with steep skirt selectivity based on stimulated Brillouin scattering", IEEE Photonics Journal, vol. 8, no. 6, pp. 5502307, December 2016.

[13] A. Zadok, E. Zilka, A. Eyal, L. Thévenaz, and M. Tur, "Vector analysis of stimulated Brillouin scattering amplification in standard single-mode fibers”, Opt. Express, vol. 16, no. 26, pp. 21692, 2008.

[14] R. W. Boyd, Nonlinear optics (Academic Press, 2008).

[15] W. Zhang, R. A. Minasian, "Widely tunable single-passband microwave photonic filter based on stimulated Brillouin scattering", IEEE Photonics Technology Letters, vol. 23, no. 21, pp. 1775, 2011. 